\title{
IMPROVING THE ACCURACY OF SELECTION OF BIRD RADAR ECHOES AGAINST A BACKGROUND OF ATOMIZED CLOUDS AND ATMOSPHERIC INHOMOGENEITIES
}

\author{
Leonid Dinevich
}

\begin{abstract}
Dinevich, L. 2015. Improving the accuracy of selection of bird radar echoes against a background of atomized clouds and atmospheric inhomogeneities. Ring 37: 3-18.

The algorithm for bird radar echo selection was developed in Israel and has been successfully used for many years to monitor birds in periods of massive intercontinental migration in order to ensure flight safety in civil and military aviation. However, it has been found that under certain meteorological conditions the bird echo selection algorithm does not filter out false signals formed by atomized clouds and atmospheric inhomogeneities. Although the algorithm is designed to identify and sift false signals, some useful echoes from smaller birds are erroneously sifted as well.

This paper presents some additional features of radar echoes reflected from atmospheric formations that can be taken into account to prevent the loss of useful bird echoes. These additional features are based on the use of polarization, fluctuation and Doppler characteristics of a reflected signal. By taking these features into account we can reduce the number of false signals and increase the accuracy of the bird echo selection algorithm. The paper presents methods for using radar echoes to identify species and sizes of birds, together with recommendations on using the data to ensure flight safety during periods of massive intercontinental bird migration.
\end{abstract}

L. Dinevich, George S. Wise Faculty of Natural Sciences, Dept. of Zoology, Tel-Aviv University, Ramat Aviv, 69978, Israel. E-mail: dinevich@013.net

Keywords: radar ornithology, accuracy, polarization 


\section{INTRODUCTION AND TASK FORMULATION}

An ornithological radar system based on MRL-5 radar enables real-time monitoring of massive intercontinental bird migration (Dinevich and Leshem, 2007; 2008; Dinevich, et al. 2004). The monitoring has two purposes. First, it is important for ornithologists to know the migration trajectories of various bird species in specific seasons and at specific times of day. It is also important to have information on large concentrations of migrating birds over different areas, as well as on the heights, directions and velocities of their flights. This information cannot be obtained by traditional observation techniques. Systematic radar data make it possible to obtain the necessary flight characteristics and to establish the correlations between migration patterns and weather conditions. Secondly, the monitoring provides real-time information allowing the airspace to be safely shared by both birds and aviation, especially military air vehicles. The ornithological situation is taken into account while planning flights.

Characteristics of radar signals reflected from various types of objects have been established and used as the basis for an algorithm that makes it possible to identify bird echoes and to plot real-time ornithological charts containing vector fields of bird movements, including distribution over height.

Ornithological radar charts plotted by means of these algorithms include the following information:

1. total number of birds currently in the air, including migrating birds

2. minimum and maximum flight velocities

3. distribution of bird echoes over height

4. spectra of velocities and directions of bird flights based on data on their echo movements, including the summary direction vector

5. vector fields of bird echo movements against the background of meteorological phenomena and ground conditions

6. distribution of bird species based on the characteristics of their echo movements (degree of straightness and uniformity)

7. information on clouds and on invisible atmospheric formations and their parameters, including their time evolution

The text is a description and discussion of problems connected with the estimation of numbers of flying birds using MRL-5 radar, but the problems are comparable when using other types of weather radar.

The radius of ornithological radar fields with respect to the radar location depends on bird sizes (Dinevich and Leshem, 2007). Generally, for flocks of birds the size of a stork this radius can reach $60 \mathrm{~km}$, while for birds the size of a sparrow it can be up to 30 or sometimes $40 \mathrm{~km}$.

Due to these factors, as well as the computational capacities of the computers used and the need to minimize the computational time required to process large data sets, the maximum exposure radius of the radar system was set at $60 \mathrm{~km}$. 
Combined radar and visual observations carried out in Moldova in the 1980s (a long-term experiment employing several MRL-5 stations) made it possible to perform reliable tracking of large flocks of storks at distances of over $100 \mathrm{~km}$ and to monitor their flights for over three hours. In the experiment, visual observations were carried out by a team from the Academy of Sciences of Moldova, led by Dr. I. Ganja. The radar research was carried out under the supervision of L. Dinevich and radar engineer V. Dinevich. (Ganja et al., 1991). The MRL-5 potential in Israel, as in Moldova, enables identification of an echo of a bird as large as a stork (e.g. cranes or pelicans) flying as high as $700 \mathrm{~m}$ at a distance of $100 \mathrm{~km}$ (Ganja et al., 1991; Dinevich et al., 2001).

Many years of work with the algorithm have shown that it can effectively distinguish bird echoes from echoes of terrestrial forms, aeroplanes, most clouds, air currents and atmospheric inhomogeneities (Dinevich and Leshem, 2007; 2008). The most problematic issue is dealing with weak and strongly fluctuating echoes from atomized clouds, dissipation zones of various cloud formations, point echoes from insects, and echoes reflected from longitudinal and hooked strips of atmospheric inhomogeneities. The nature of such inhomogeneities has been well analysed by Chernikov (1979).

In the presence of these atmospheric inhomogeneities, false signals (noise) often appear on the ornithological charts plotted by the bird selection algorithm.

\section{EXAMPLES OF FALSE SIGNALS IDENTIFIED BY A RADAR SYSTEM AS BIRD ECHOES}

Examples of problems with accurate bird selection are given in Figures 1-5, which are ornithological charts plotted on different dates as standard output from data processing. The charts are commented on below, as in some cases the vectors identified as birds are created by false echoes, i.e. echoes not related to birds.

In Figure 1 we can see echoes from clouds, precipitation, hills and ground clutter. The exposure radius is over $50 \mathrm{~km}$. The colours in the lower right-hand corner of the chart designate the levels of radar echo. The weather conditions when the chart was plotted are shown together with the birds: convective cloud formations, discrete showers and moderate north-west winds. The clouds are slowly moving towards the south-east. In such atmospheric conditions, there may be only a few birds in the air.

Figure 2 presents the same data as in Figure 1, but after selection of radar echoes by the bird recognition algorithm. In this case, the selection parameters are set to the supersensitive level. The algorithm filters processed the echoes and plotted the vectors in the border areas or dissipation areas of the clouds. It is reasonable to assume that only some of the vectors are related to bird echoes.

Figure 3 presents the same data as in Figure 1 after selection of bird echoes performed at a less sensitive level. The ornithological chart features far fewer vectors than in Figure 2, thus reflecting the actual ornithological situation more realistically. In order to obtain this chart, we had to set the selection parameters at a lower sensitivity. Consequently, it is very likely that we lost some echoes from smaller birds. Hence the number of birds can be over- or underestimated depending on the parameters of selection. 


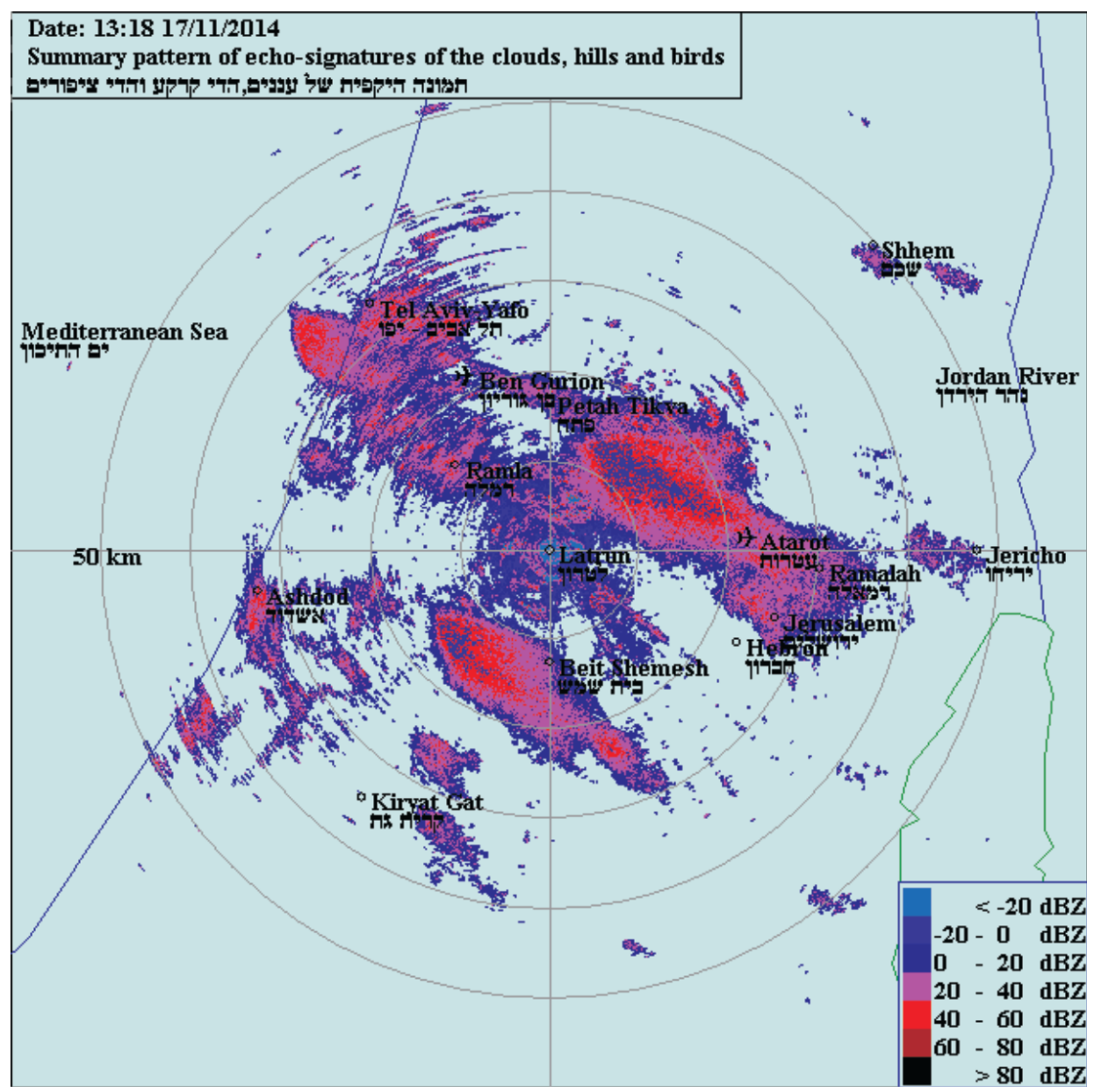

Fig. 1. Echoes from clouds, precipitation, hills and ground clutter. The exposure radius is over $50 \mathrm{~km}$. The colours in the lower right-hand corner of the chart designate the levels of radar echo. The weakest echo level is indicated by the colour blue.

In Figures 4 and 5, the vectors show the daytime movements of birds. Most of the vectors are either scattered all over the chart or form long strips; they mainly have distinct directions of bird migration for a given corresponding season.

In both charts (Fig. 4-5), the areas designated as '1', '2' and ' 3 ' are characterized by a set of vectors which, unlike the others, are chaotically oriented and have a higher concentration per unit volume. Such areas, as a rule, correspond to visually observable weak convective clouds or stratus cloud formations. Most often, these areas form within the sub-inversion layer (1-1.5 km), but sometimes even lower due to the high gradients of temperature and humidity. In Israel, such atmospheric micro-fronts emerge in the valleys between hills and at the land and sea borders. The features used in the algorithm receive signals from zones of strongly fluctuating weak echoes reflected from clouds and interpret them as bird echoes, thus plotting false vectors. 


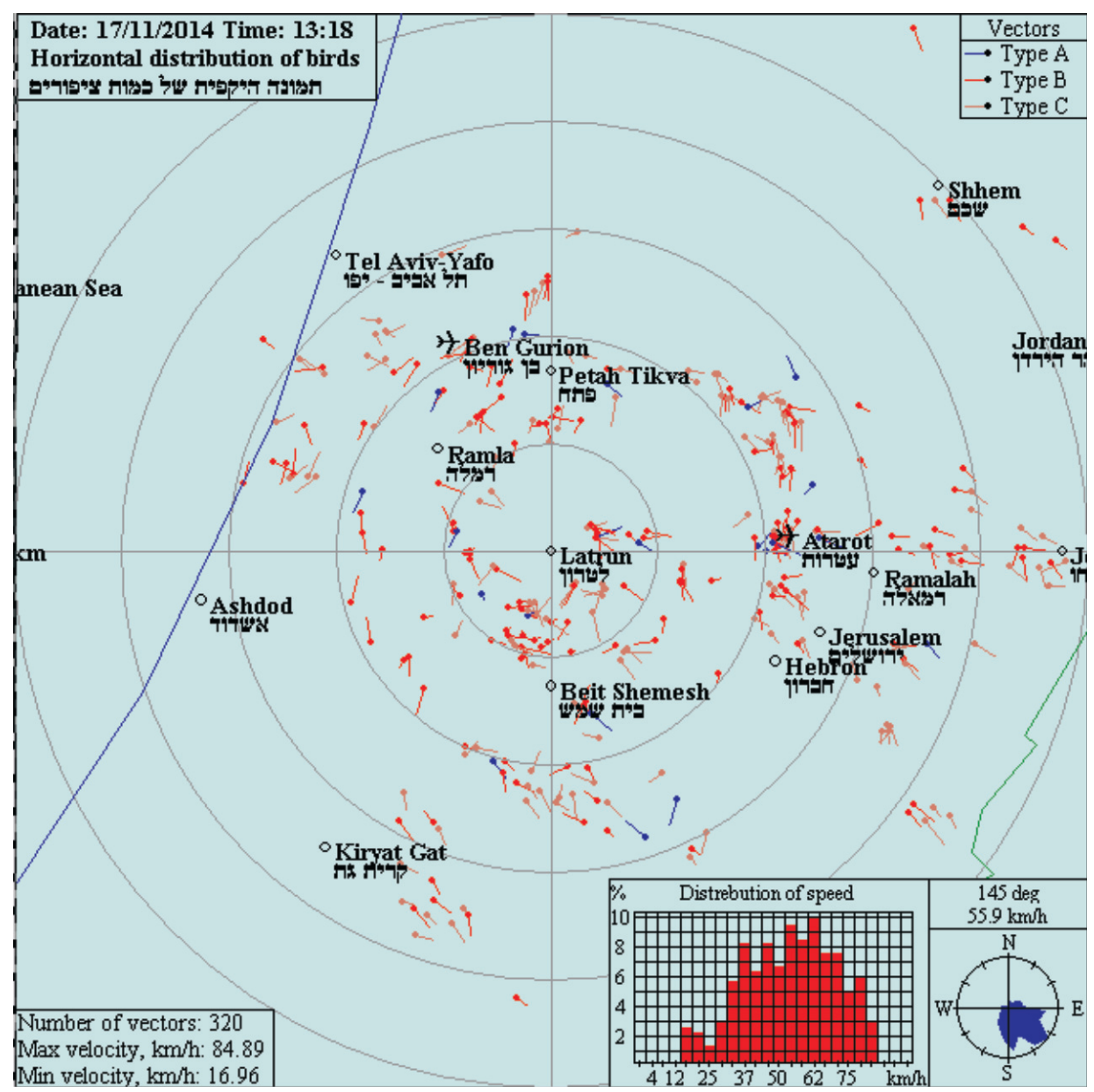

Fig. 2. The same chart as in Fig. 1 after selection of radar echoes by the bird recognition algorithm. In this case, the selection parameters are set at the supersensitive level.

A characteristic feature of such vector formations is their high density and chaotic direction. The algorithm makes it possible to distinguish them. However, as shown above in the discussion of Figures 1-3, this requires reducing the method's sensitivity and, as a natural consequence, may lead to the loss of some useful information. Similar problematic zones often emerge in areas of visible and invisible atmospheric formations.

Figure 6 shows examples of radar echoes from invisible atmospheric inhomogeneities often forming in Israel within the lower sub-inversion level at the height of $1-1.5 \mathrm{~km}$, as well as in valleys in the early morning hours. One can see large and small compact spots formed by echoes from hills. Strips of different lengths, sometimes forming non-straight lines are most often formed by echoes from invisible atmospheric formations. 


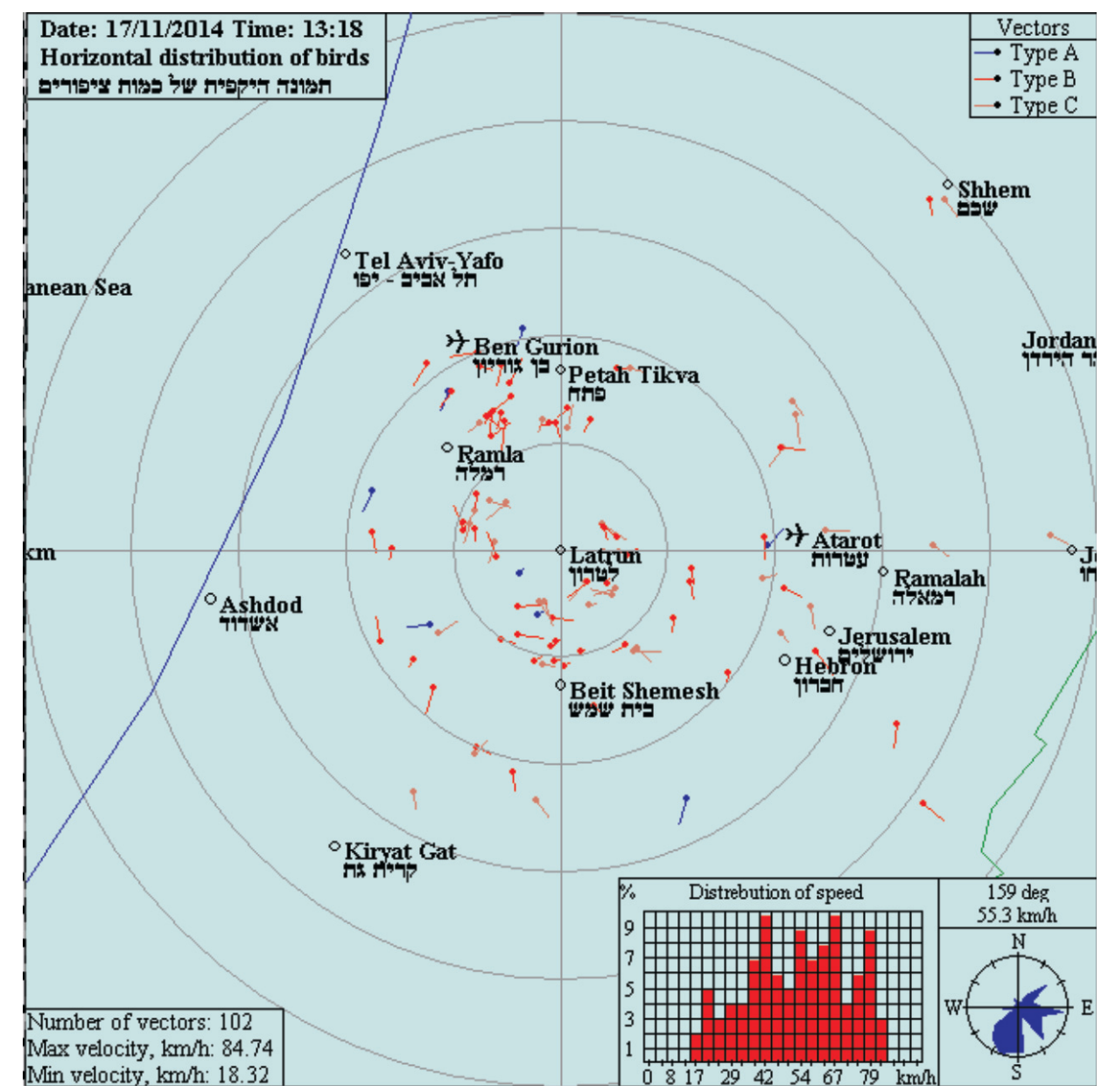

Fig. 3. The same chart as in Fig. 1 after selection of bird echoes performed at a less sensitive level

In a book by Anon. (1993), in Section 2.10.8.2. 'Anomalous radar echoes in the form of thin lines and strips', the author explains the following:

1. Radar echoes of this type move ahead of intensive thunderstorms and are related to the wind shift. In the area of the shift, one often observes increasing concentrations of dust particles and pollen, as well as a sharp increase in the refraction coefficient gradient.

2. Many researchers have observed thin lines in the absence of clouds and related them to wind shift zones. Such zones often emerge in coastal areas and are considered to be related to breezes. The radius of detection of the thin lines in these cases seldom exceeds $50 \mathrm{~km}$, and the height is not over 1-2 km.

3. Radar echoes in the form of strips within a radius of up to $40 \mathrm{~km}$ may be reflected from cirrus clouds located much higher than the levels of bird flights. 


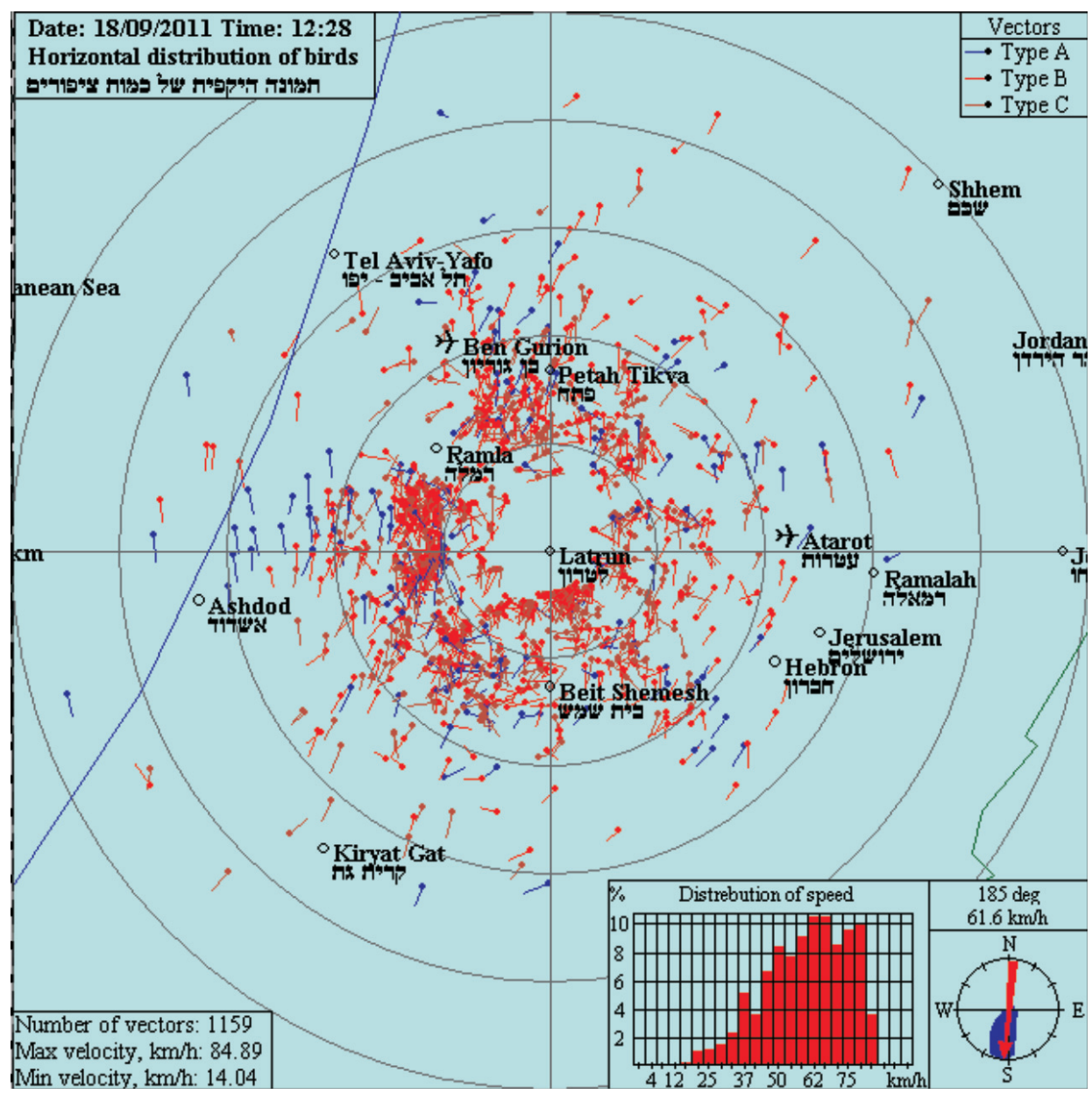

Fig. 4. Example of vectors representing the daytime movements of birds. Further explanations in the text.

4. In addition, echoes in the form of long strips may be reflected in the early morning from inhomogeneities emerging due to higher humidity between hills. The inhomogeneities form either as a result of the wind draught in the humid sea air, or to the morning bottom layer convection of the air cooled between hills during the night. As a rule, these echo strips disappear as the sun warms the bottom atmospheric layers.

Here, as in the examples discussed earlier (Figures 1-3), the features used in the algorithm interpret echo signals as bird echoes and form false vectors. For such situations there is a typical high concentration and chaotic distribution of vectors. The algorithm described by Dinevich and Leshem $(2007,2008)$ is able to distinguish between false vectors and vectors formed by bird movements. However, as follows from the explanations of Figures 1-3, this requires reducing the method's sensitivity, thereby losing some useful echoes. 


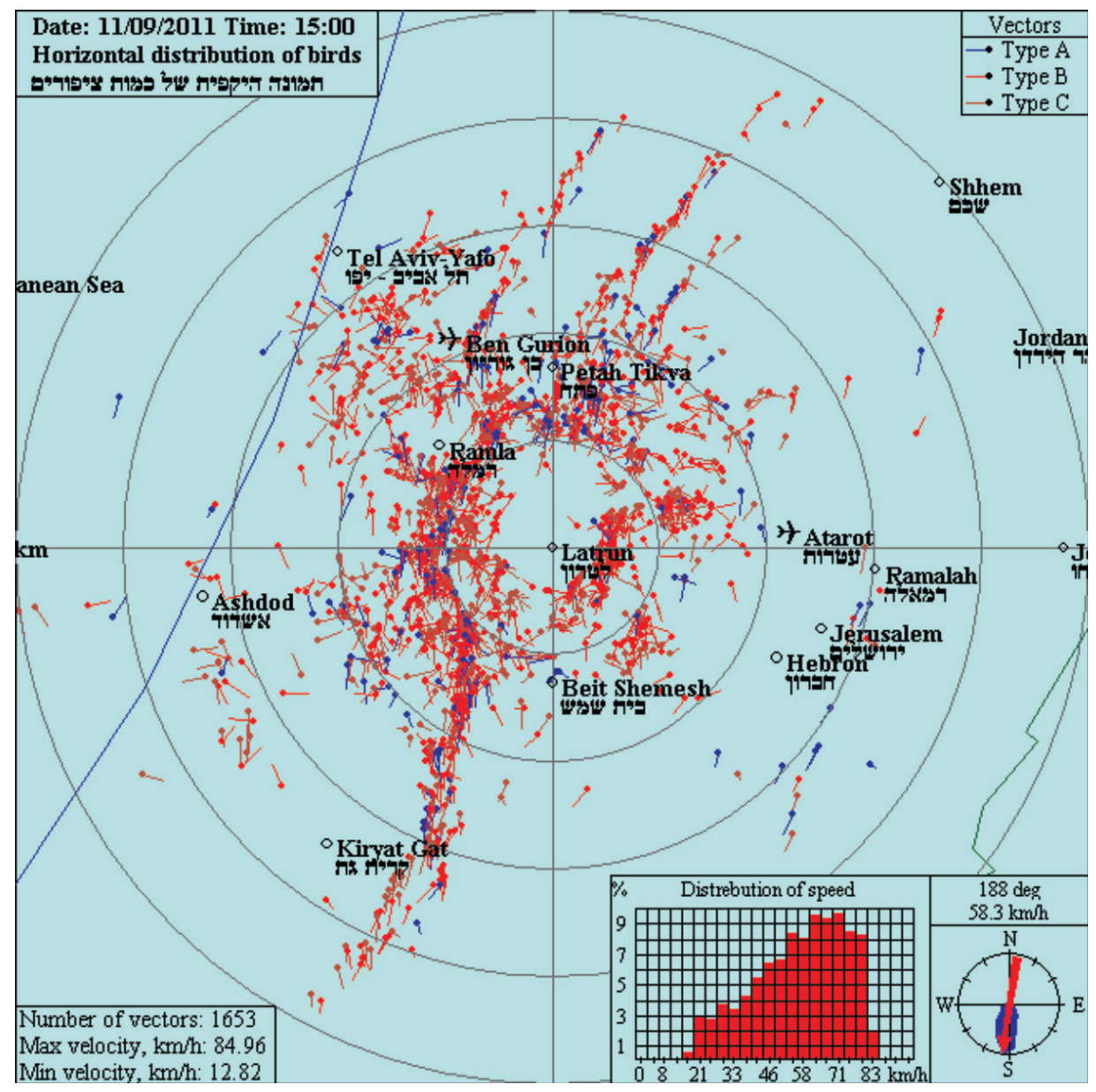

Fig. 5. Example of vectors representing the daytime movements of birds. Further explanations in the text.

False signals are often formed within the near-field zone by echoes from radar lobes (Fig. 7). This figure shows a vertical section of a large bird flock in the daytime. The exposure radius is $50 \mathrm{~km}$, and the total length of the flock is over $100 \mathrm{~km}$. On the screen, only half of the length in one direction can be seen. The height of the echo is $800-1200 \mathrm{~m}$. The maximum echo concentration is at a height of $800 \mathrm{~m}$. Bird echoes look like vertically stretched short strips, with the length of the strips dependent on the value of the antenna diagram and on the echo power, i.e. on the reflection power of the target. Within the same diagram, the length of an echo strip reflected from a single stork or a stork flock would be different from that of a Passeriformes flock. Certain weak echoes in the near-field zone at a height significantly over 1,200 m are worthy of note: these are echoes from ground clutter formed by the radar lobe and erroneously interpreted by the algorithm as bird echoes. 


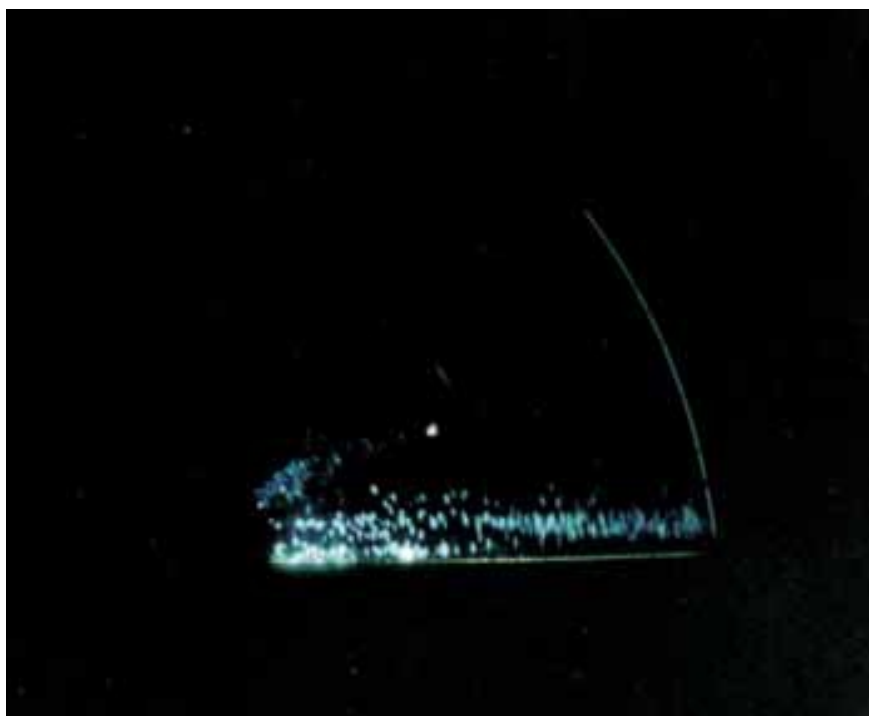

Fig. 7. False signals formed within the near-field zone

\section{POLARIZATION CHARACTERISTICS OF RADAR ECHO}

According to the basic equation of radiolocation (Dinevich and Kaplan, 1999), the distance at which a single target can be identified increases with an increase in transmitter power and antenna power gain, with decreasing intrinsic noise of the radar station receiver and increasing $\sigma$ of the target.

At calculated values of a distance $R$ to the target and the power of the reflected signal $P \tau$, one can calculate the value of the effective scattering area (ESA) $\sigma$ of a single target.

$$
\sigma=\frac{(4 \pi)^{3} P r R^{4}}{P \tau G^{2} \lambda^{2}} \frac{1}{K_{1}} \frac{1}{K_{2}}
$$

This formula can be used to calculate the ESA of a single bird, while the values of $K_{1}$ and $K_{2}$ in the absence of atmospheric precipitation can be assumed equal to unity. According to the physical meaning of ESA, the larger the bird, the higher the value of $\sigma$ is.

In studies by Shupijatcky (1959) and Chernikov and Schupjatsky (1967), ESA of birds was found to be distinctly dependent on polarization of the signal (both transmitted and received) and on the bird's orientation with respect to the radar emitter. The extent of depolarization found by the authors is about -7 to $-9 \mathrm{~dB}$. For a polarimeter with linear polarization of emission and receipt, depolarization is defined as the proportion between the orthogonal component and the basic component of an echo signal. If the emitted waves have a horizontal polarization, the polarization expression can be written in the following form:

$$
\Delta P x=P x y / P x x \text { or } \Delta P x(d B)=\lg P x y / P x x
$$




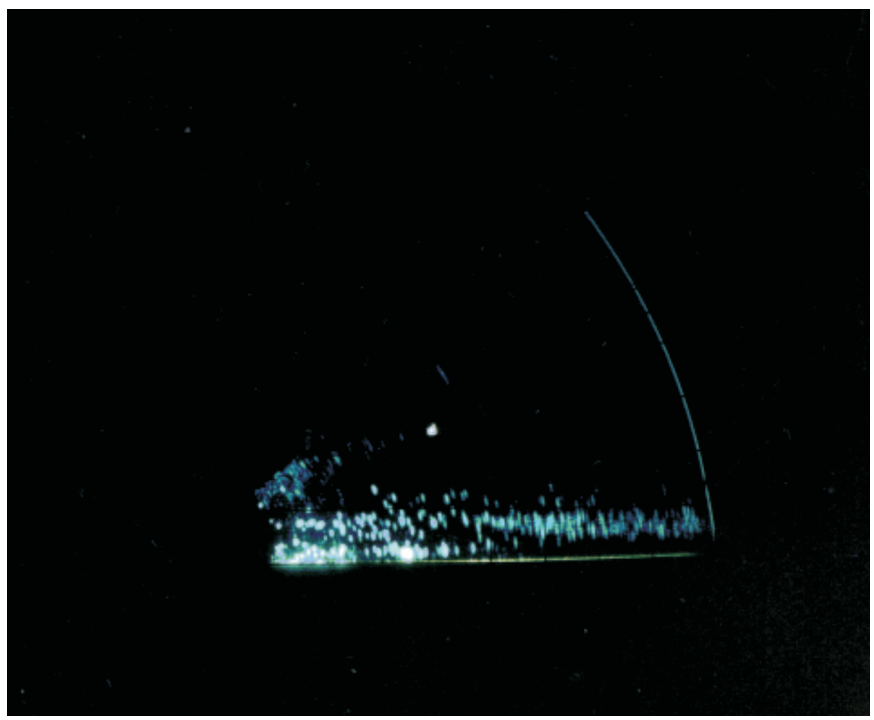

Fig. 7. False signals formed within the near-field zone

\section{POLARIZATION CHARACTERISTICS OF RADAR ECHO}

According to the basic equation of radiolocation (Dinevich and Kaplan, 1999), the distance at which a single target can be identified increases with an increase in transmitter power and antenna power gain, with decreasing intrinsic noise of the radar station receiver and increasing of of the target.

At calculated values of a distance $R$ to the target and the power of the reflected signal $P \tau$, one can calculate the value of the effective scattering area (ESA) $\sigma$ of a single target.

\section{[wzór]}

This formula can be used to calculate the ESA of a single bird, while the values of $K_{1}$ and $K_{2}$ in the absence of atmospheric precipitation can be assumed equal to unity. According to the physical meaning of ESA, the larger the bird, the higher the value of $\sigma$ is.

In studies by Shupijatcky (1959) and Chernikov and Schupjatsky (1967), ESA of birds was found to be distinctly dependent on polarization of the signal (both transmitted and received) and on the bird's orientation with respect to the radar emitter. The extent of depolarization found by the authors is about -7 to $-9 \mathrm{~dB}$. For a polarimeter with linear polarization of emission and receipt, depolarization is defined as the proportion between the orthogonal component and the basic component of an echo signal. If the emitted waves have a horizontal polarization, the polarization expression can be written in the following form:

$$
\Delta P x=P x y / P x x \text { or } \Delta P x(d B)=\lg P x y / P x x
$$


In the case of a vertical polarization of the emitted wave, the polarization expression is $\Delta P y=P y x / P y y$. Here $P x x, P y y, P x y$ and $P y x$ are components of the echo signal power, the first index is related to the type of polarization at emission, and the second index stands for the type of polarization at reception. $x$ and $y$ stand for horizontal and vertical polarization, respectively.

In the case of a pulse-wise commutation between emission polarization and receipt of signal at the same polarization, one can calculate the value of the differential reflectivity as follows:

$$
d P=P x x / P y y \text { or } d P=10 \lg P x x / P y y
$$

Several studies (Shupijatcky, 1959; Dinevich, 1981; Dinevich et al. 1990, 1994) have shown that the values of depolarization and of the differential polarization are functionally related only to the shape of the target and its orientation in space, and do not depend on any other parameters, including the dielectric conductivity of a body or signal attenuation along the route. Using the two polarization components $\triangle P x$ and $d P$ one can calculate the orientation of birds in space as well as their shape (i.e. the ratio of the length to the width).

The formula for calculation of the angle of a bird's orientation in space (Shupijatcky, 1959) is as follows:

$$
\operatorname{tg} 2 \theta=2 d P^{1 / 2} \Delta P^{1 / 2}\left[d P^{1 / 2}-1\right]
$$

where $\theta$ is the angle of a bird's orientation in space.

Another important factor is the ability to use the value of the differential reflectivity in order to isolate signals from birds on the background of echoes from various atmospheric inhomogeneities whose nature has nothing to do with emergence of visible hydrometeors. The differential reflectivity of such inhomogeneities is close to unity, while the differential reflectivity of birds is much higher than unity. This means that this feature of an echo can be used to filter out signals reflected from these inhomogeneities.

Our research (Dinevich et al. 1994) shows that the differential reflectivity of atomized clouds tends to unity. This can be easily demonstrated by a simple example. By definition, the differential reflectivity $d P=10 \mathrm{lg} P x x / P y y$. Small drops up to $0.5 \mathrm{~cm}$ always have a spherical shape (Litvinov, 1974; Minervin et al., 1971), i.e. the values of the radiuses of the mutually perpendicular axes are close. Therefore, $P x x \approx P y y$. Hence $P x x / P y y \approx 1$ and, consequently, $d P \approx 1$.

According to observations (Dinevich et al., 1994), radar reflectivity of atomized clouds does not exceed $30 \mathrm{dBZ}$, which is also the extreme level of reflectivity of birds.

Therefore, signals with parameters $\leq 30 d B Z$ and $d P \approx 1$ typical of reflections from small drops are filtered out, while signals with parameters $\leq 30 d B Z$ and $d P>1$, together with other signal characteristics (fluctuation, mobility in space, etc.) might be those reflected from birds. This approach enables observation of birds against the background of atomized clouds.

Another important characteristic of signals making it possible to distinguish reflections from insects and various suspended substances in the atmosphere (plant seeds, dust particles, etc.) from bird echoes, is a complex parameter including two 
signal components, i.e. the value of $\sigma$ and its depolarization $\Delta P x=P x y / P x x$ or $\Delta P x$ $(d B)=\lg P x y / P x x$.

It is typical of signals reflected from birds and insects to have a mean depolarization of 11-13 $d B$ (Shupijatcky, 1959). However, this value for birds exceeds $10^{-1} \mathrm{~cm}^{-1}$, while for insects it is significantly lower.

An important advantage of using this parameter is the fact that at equal exposure volumes the polarization relationships do not depend on the concentration of targets within the volumes. At the same time, the consistency of dispersion does depend on the concentration (multiple targets vs. single target). This condition is highly important for bird echo selection within a zone of atomized clouds. The echo from largedrop clouds is easily recognized by the algorithm described above. Irrespective of the number of birds in the air, they always have a certain space orientation. Small spherical drops do not have such orientation and thus can be easily identified on the basis presented above.

\section{CALCULATION OF BIRD NUMBERS AND DETERMINATION OF THEIR SPECIES AND SIZE}

Selection of bird echoes on a background of other reflecting objects at a rate close to high reliability enables the next stage of calculation of bird quantities and determination of their species and size.

A study by Dinevich and Leshem (2007) presents an evaluation of the dependence of the probability of bird detection at distance $R$ on the damping of signals. The evaluation was performed experimentally, in a way similar to that of issuing a storm alarm in case of showers and thunderstorms. For these meteorological situations, on the basis of very rich data, the following values were obtained on the probability of detecting phenomena while cumulonimbus clouds $(\mathrm{Cb})$ occur with showers and thunderstorms.

Table 1

Probability (in \%) of detecting weather phenomena in different weather situations.

The data were obtained by means of the MRL with respect to the distance.

\begin{tabular}{|c|r|r|r|r|r|r|r|}
\hline & \multicolumn{7}{|c|}{ Distance from the radar, km } \\
\cline { 2 - 8 } & 0 & 50 & 100 & 150 & 200 & 250 & 300 \\
\hline With thunderstorm & 100 & 100 & 100 & 100 & 85 & 40 & 5 \\
\hline With showers & 100 & 100 & 95 & 70 & 45 & 15 & - \\
\hline
\end{tabular}

If we assume that the radius $R$ reliable for the detection is the one that enables a high probability of detecting the abovementioned meteorological situations (with reliability not less than $90-100 \%$ ), it follows from the table that the corresponding value of $R$ for MRL is $90-100 \mathrm{~km}$. This means that at these distances MRL does not 'miss' any of the dangerous phenomena. The problem of bird echo identification has been solved in a similar way. However, in the case of bird identification it was necessary to take into account the coefficient related to the enhancement of the volume 
of the radar exposure while shifting from the experimental distance of $100 \%$ detection to actual distances of $20,30,40,50,60$, etc. km. For instance, the following considerations were made for the calculation algorithms.

1. Watching the MRL-5 screens, we determine the distance at which no bird echoes were seen. For example, in 53 photos the extreme distance ranges between 45 and $60 \mathrm{~km}$ within the azimuth sector where there are no take-off angles. One may assume that while birds are flying they fill this sector beyond that radar exposure area. Therefore, at $100 \%$ probability, in all 53 cases you see the birds at distances of up to $45 \mathrm{~km}$.

2. Assume that we have established that at distances of $45-60 \mathrm{~km} 173$ bird echoes were identified in all 53 cases. In this case, the following table (Table 2) can be made:

Table 2

Probability of bird echo identification at different distances

\begin{tabular}{|l|c|c|c|}
\hline \multirow{2}{*}{} & \multicolumn{3}{|c|}{ Distances, km } \\
\cline { 2 - 4 } & $45-50$ & $51-55$ & $56-60$ \\
\hline Number of bird echoes & 95 & 50 & 30 \\
\hline$\%$ & 54.3 & 28.6 & 17.1 \\
\hline \multicolumn{2}{|c|}{ Total identification of 175 echoes $=100 \%$} \\
\hline
\end{tabular}

For different bird species, tables of this kind should differ. If no species differentiation is made, a general evaluation of the probability can be performed by this method.

It is necessary to introduce a coefficient that takes into account the increase in volume while shifting from the $20 \mathrm{~km}$ distance (the distance enabling $100 \%$ identification of all the birds) to distances of $30,40,50,60 \mathrm{~km}$, etc.

In cases when the radar is supposed to provide the aviation services with realtime information on the precise locations of birds in space and time, two kinds of ornithological charts must be calculated: a) a chart of actual locations of echo vectors and b) a chart based on calculations of probabilistic locations of echoes, taking into account the considerations given above.

The precision of detecting birds by means of MRL-5 can be evaluated using the equation for radiolocation of a pinpoint target:

$$
P_{r}=\frac{1.46 \cdot P_{t} \cdot G^{2} \cdot l^{2} \cdot \sigma}{(4 \pi)^{3} L \cdot R^{4} w}
$$

where $\mathrm{s}$ is the effective scattering area (ESA) of the target, 1 is the wavelength, $P_{t}$ is the radiation pulse power, $G$ is the antenna gain, $L(\mathrm{~dB})$ is the damping in the transceiving tract (in both directions) and $R$ is the distance to the target.

Taking into account the dimensions of MRL parameters, the expression (1) will have the following form:

$$
P_{r}[\mathrm{~W}]=\frac{7.4 \cdot 10^{-13} \cdot P_{t}[\mathrm{~kW}] \cdot G^{2} \cdot \lambda^{2}[\mathrm{~m}] \cdot \sigma\left[\mathrm{m}^{2}\right]}{R^{4}[\mathrm{~km}] \cdot L}
$$

where $7.4 \cdot 10^{-13}$ is a coefficient taking into account the dependence $1.46 /(4 \pi)^{3}$ (from formula (1)). 
The ESA of the target is established as follows:

$$
\sigma\left[\mathrm{m}^{2}\right]=\frac{P_{r \min }[\mathrm{W}] \cdot 10^{0.1 \mathrm{n}} \cdot 10^{12} \cdot R^{4}[\mathrm{~km}]}{1.46 \cdot P_{t}\left[\mathrm{~kW}_{\mathrm{T}}\right] \cdot 10^{3} \cdot G^{2} \cdot \lambda^{2}[\mathrm{~m}] \cdot \eta_{\mathrm{rec}} \cdot \eta_{\mathrm{tr}}}
$$

where $\eta_{\text {rec }}$ and $\eta_{\text {tr }}$ are the values of the efficiency factor for the radar station receiver and transmitter, respectively. We always assumed those values to be equal to unity.

From equation (2), based on the parameters of a specific radar station (here we entered the parameters of our MRL-5), one can obtain a dependence in the form of $\left.\sigma_{\min }\left[\mathrm{m}^{2}\right]=\varphi R[\mathrm{~km}]\right)$. This dependence enables evaluation of the minimum ESA value $\left(\sigma_{\min }\right)$ of a target bird that makes it possible for MRL-5 to identify this target at a given distance $R$.

$$
\begin{aligned}
& \text { For MRL-5 at } \lambda_{1}=3.14 \mathrm{~cm} \rightarrow \sigma_{\min }\left[\mathrm{m}^{2}\right]=2.0 \cdot 10^{-10} R^{4}[\mathrm{~km}] \cdot 10^{0.1 \mathrm{n}} \\
& \text { at } \lambda_{2}=10.15 \mathrm{~cm} \rightarrow \sigma_{\min }\left[\mathrm{m}^{2}\right]=1,3 \cdot 10^{-10} \mathrm{R}^{4}[\mathrm{~km}] \cdot 10^{0.1 \mathrm{n}}
\end{aligned}
$$

Here the multiplier $10^{0.1 \mathrm{n}}$ takes into account the excess in the useful signal over the intrinsic noises of the receiver. For example, if the excess $n(\mathrm{~dB})$ is

$$
n=7 \mathrm{~dB} \text {, then } 10^{0.1 \mathrm{n}}=10^{0.1 \cdot 7}=10^{0.7}=\underline{5.01} \text {. }
$$

In this case, the ESA of the bird at $\lambda_{1}=3.14 \mathrm{~cm}$ is

$$
\sigma\left[\mathrm{m}^{2}\right]=2.0 \cdot 10^{-10} R^{4}[\mathrm{~km}] \cdot \underline{5.01} .
$$

For example, the maximum distance at which you can detect birds when the damping is not introduced into the receiving tract (i.e. $10^{0.1 \mathrm{n}}=10^{0,1 \cdot 0}=10^{0}=1$ ) is $45 \mathrm{~km}$.

For MRL-5 at:

$$
\lambda_{1}=3.14 \mathrm{~cm}, \quad \sigma_{\min }\left[\mathrm{m}^{2}\right]=2.0 \cdot 10^{-10} \cdot \mathrm{R}^{4} \cdot 10^{0.1 \mathrm{n}},
$$

where ESA $\left(\sigma_{\min }\right)$ is the minimum value required for detecting birds at different distances. At $R=45 \mathrm{~km}\left(R^{4}=454=4.1 \cdot 10^{6}\right)$.

Therefore, at $R=45 \mathrm{~km}$ MRL-5 at $\lambda_{1}=3.14 \mathrm{~cm}$ detects birds if from:

$$
\sigma_{\min }=2.0 \cdot 10^{-10} \cdot \mathrm{R}^{4}=2.0 \cdot 10^{-10} \cdot 4.1 \cdot 10^{6}=8.2 \cdot 10^{-4} \mathrm{~m}^{2},
$$

i.e. if we see a slight track of a bird echo on the MRL screen, the ESA of the bird is approximately equal to $8.2 \cdot 10^{-4} \mathrm{~m}^{2}$. In this way, even without introducing the damping into the receiving tract, one can evaluate the ESA of a bird.

At $\sigma_{\min } 8.2 \cdot 10^{-4} \mathrm{~m}^{2}$ and at $R=45 \mathrm{~km}$, MRL-5 does not detect birds.

If at $R=45 \mathrm{~km}$ birds are identified, but then their images disappear from the screen when values such as $12 \mathrm{~dB}$ (i.e. $10^{0.1 \mathrm{n}}=10^{1,2}=1.58 \cdot 10^{1}$ ) are introduced into the receiving tract, this means that at this distance:

$$
\sigma_{\min }=8.2 \cdot 10^{-4} \cdot 1.58 \cdot 10^{1}=12.9 \cdot 10^{-3}=1.3-10^{-2} \mathrm{~m}^{2} ;
$$

therefore the ESA of a bird is not less than $1.3 \cdot 10^{-2} \mathrm{~m}^{2}$.

If a bird is regarded as an ellipsoid of revolution, the two polarization components $\Delta \mathrm{Px}$ and $d P$ will enable calculation of the ratio of axes a and $\mathrm{b}$ (see above). Knowing the ESA $(\sigma)$ and the ratio of the bird's axes, one can establish its species and size. 


\section{SOME CONSIDERATIONS CONCERNING OBTAINING AND IMPLEMENTING POLARIZATION CHARACTERISTICS OF RADAR ECHOES AIMED AT IMPROVING THE ACCURACY OF BIRD ECHO SELECTION}

According to calculated and experimental evaluations (Dinevich, 1981; Chernikov and Schupjatsky, 1967), in order to calculate the depolarization characteristics and differential polarization of a radar echo, the orthogonal characteristics of the echo should be averaged over at least 32 pulses.

MRL-5 emits 500 pulses per second. The antenna beam width at $\lambda_{1}=3.14 \mathrm{~cm}$ is 0.5 degrees, and at $\lambda_{2}=10 \mathrm{~cm} 1.5$ degrees.

Consequently, in order to obtain at least 32 reflected pulses, the antenna frequency should not exceed one pulse per second. At this frequency, it will take the antenna $\approx 0.14 \mathrm{sec}\left(60 \mathrm{sec} / 360^{\circ} \approx 0.14 \mathrm{sec}\right)$ to scan a sector of one degree. For a width of 1.5 degrees $\left(\lambda_{2}=10 \mathrm{~cm}\right)$ the number of pulses will be 1.5 times higher.

If the data needed to assess the ornithological situation are obtained from 5 levels (e.g. for the 1.5 degree beam the vertical antenna angles can be set at $0^{\circ}, 0.5^{\circ}, 1.0^{\circ}$, $1.5^{\circ}, 2.5^{\circ}, 3.5^{\circ}, 4.5^{\circ}$ and $5.5^{\circ}$ ), the time needed for the data collection is $8 \mathrm{~min}$, or $480 \mathrm{sec}$.

With the algorithm currently available, it will take over 10 minutes to collect the data from this number of levels at a frequency of 6 revolutions per minute $(10 \mathrm{sec}$ per revolution $\times 8$ revolutions $\times 5-8$ levels $=400$ to $640 \mathrm{sec}$ ). The expected accuracy of bird echo selection if the polarization characteristics are implemented is higher than the accuracy of the algorithm used today. However, this method does not allow for plotting of movement vectors. Therefore, both selection methods should be used. Vector ornithological charts illustrate bird movement dynamics, while the polarization method enables evaluation of the precise number of birds in the air. In addition, by calculating parameters of a particular echo, we can determine the bird's size and species.

\section{CONCLUSIONS}

The considerations presented above show the possibility of improving the accuracy of bird echo selection through implementation of newly found additional features complementing the existing algorithm developed in Israel. This implementation requires special equipment for pulse-wise emission of electromagnetic energy, in both the horizontal and vertical directions, which is able to properly receive the orthogonal components. The frequency isolation between the two channels should not be below $28 \mathrm{~dB}$. This level of isolation was provided by the equipment used by the author while developing the method for hail detection in clouds (Dinevich, 1981). Using both methods in succession will make it possible not only to increase the accuracy of bird echo selection and to establish bird size and species, but also to plot vector ornithological charts for different levels and all over the specified volume at a radius of $60 \mathrm{~km}$ in the daytime and $40 \mathrm{~km}$ at night. 
As a result, the method will make it possible to plot different types of ornithological charts, including the following:

- 3D ornithological charts of vector distribution of birds over squares and heights

- similar probability ornithological charts of vector distribution of birds, interpolated over larger distances from the radar

- ornithological charts free of false signals with calculated values of ESA $(\sigma)$, and as a result, with evaluations of the sizes and species of birds

\section{ACKNOWLEDGEMENTS}

The author expresses his sincere gratitude to Prof. Y. Leshem, a colleague in the development of bird radar location and a co-author of most academic publications on the subject, and to the Administration of Tel Aviv University and the Society for Nature Protection in Israel for the opportunity to carry out research in the field.

\section{REFERENCES}

Ganja I., Zubkov M., Kotjazi M. 1991. Radar ornithology, Stiinza, pp. 123-145.

Chernikov A. 1979. Radar clear sky echoes. Leningrad, Hydrometeoizdat, 3-40.

Chernikov A., Schupjatsky A. 1967. Polarization characteristics of radar clear sky echoes. Transactions of USSR academy of sciences, atmosphere and ocean physics, 3, 2: 136-143.

Dinevich L. 1981. Certain Features of the Structure and Evolution of Hail Producing Cumulonimbus Clouds. J. Meteorological and Hydrological, 1981, pp. 41-49.

Dinevich L., Kapitalchuk I., Schupjatsky A. 1990. Measurement of the microphisical characteristics of clouds and precipitation with a dual - polarization radar. Artificial modification of atmospheric processes in Moldova, iss 2, Kiscineu.

Dinevich L., Kapitalchuk I., Schupjatsky A. 1994. Use of the polarization selection of radar signals for remote sounding of clouds and precipitation. 34th Israel Annual conference on Aerospace science, pp. 273-277.

Dinevich L., Kaplan L. 1999. On radar observation of birds migrations. J. Scientific Israel - Technological Advantages 4.

Dinevich L, Leshem Y., Sikora O. 2001. Radar observations analysis of season bird migration in Israel at night (Based on data of radar photo registration obtained in 1998-2000), J. Scientific Israel - Technological Advantages, 3, 1-2.

Dinevich L., Leshem Y. 2007. Algorithmic system for identifying bird radio-Echo and plotting radar ornithological charts. Ring 29, 1-2: 3-39.

Dinevich L., Leshem Y. 2008. Identification of migrating birds' echo and plotting ornithological charts based on MRL-5 radar data. Advanced Contemporary Radioelectronics 3: 48 -68, The Institute for Radio Engineering and Radioelectronics, Russian Academy of Science, Moscow.

Dinevich L. 2009. Radar Monitoring of Bird Migration. Tel Aviv University, pp. 172.

Litvinov I. 1974. Structure of atmosfernych osadkov. L. Gidrometeoizdat.

Minervin W., Morgunov C., Schupiatsky A. 1971. Polarizacionnye isledovaniya stukturi kucevodozdhevich oblakov. Trudy CAO, 95.

Rukovodstvo po nabliudenii i primienieniyu informacyi s nieavtomatizovannich radiolokatorov MRL-1, MRL-2, MRL-5 (RD 52.04.320-91), S-Pb, Gidrometeoizdat.

Schupjatsky A. 1959. Radiolokacionnye razseiannie niesfericeskimi casticami. Trudy CAO, 30, pp. 39-52. 